\title{
Application of PHM for Complex Electromechanical Equipments
}

\author{
Minghui Wu, Tianzhu Wen, Shang Meng, Haomin Dai \\ Department of Scientific Research \\ Naval Aeronautical and Astronautical University \\ Yantai China \\ E-mail: minghuiwu6@sina.com
}

\begin{abstract}
PHM (Prognostic and Health Management) which is proposed by American army in JSF plan has gone through the fault diagnosis, fault prognostic and system integration three phases, and develops fast in both electronic and mechanical field. In this paper, the technical superiority of PHM is analyzed, and the framework of PHM for complex electromechanical equipment is built, which contains state monitoring, information processing and health management. Furthermore, the PHM system for complex electromechanical equipment is designed. At last, its application for DTG (Dynamically Tuned Gyroscope) shows that the system is feasible and usable.
\end{abstract}

Keywords-PHM, electromechanical equipments, state monitoring, information processing, decision making

\section{INTRODUCTION}

PHM (Prognostic and Health Management) is one of key technologies of autonomic logistics system for JSF (Joint Strike Fighter) [1]. Fault prognostic is used to determine the remaining life or normal working hours of parts. Health management is a kind of decision making capability according to diagnosis/prognostic information, available resources and use requirement. PHM make condition-based maintenance substitute for event-driven reactive maintenance and time-related preventive maintenance. Furthermore, PHM can supply precisely maintenance for accurate parts at exact time, so that the reliability is increased and the security cost is reduced.

Due to the structure and function increasing complexity, the security and stability demands of electromechanical equipment are higher and higher. So the application of PHM has important practical and theoretical significance for complex electromechanical equipment researches.

\section{PHM ADVANTAGE ANALYSIS}

As a bond technology between equipment and its support device, PHM can be embedded into the whole life cycle from development, production, experiment, use, maintenance, support to scrap. And it has significant advantages in improving the safety, reliability, maintainability, supportability and testability of the equipment [2], which are depicted in Fig.1.

1) Safety: In traditional fault diagnosis, the fault can only be identified after happening, but PHM can forecast the fault of device and subsystem and supply/provide enough time to improve system safety, so that the system-level fault and catastrophic fault can be avoided.

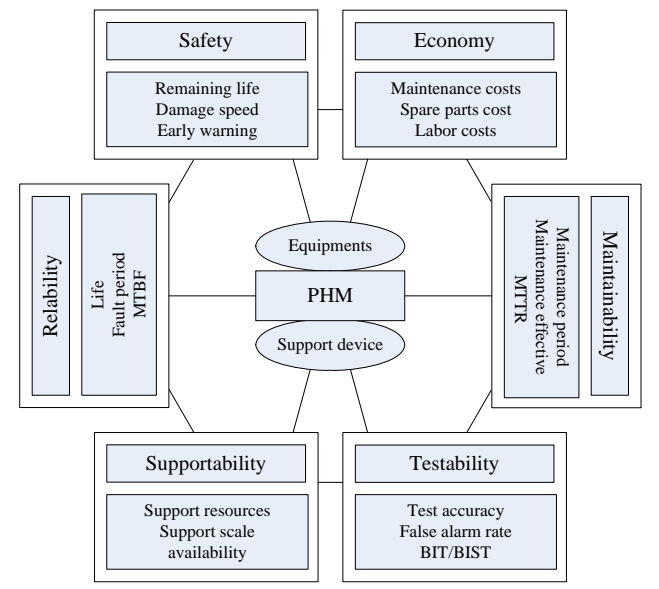

Figure 1. Advantages of PHM

2) Reliability: PHM can real-time monitor environment and running load (temperature, humidity and voltage), which affect system life, by advanced sensors. It takes proactive ways to prolong service life and fault period, thus improving overall reliability.

3) Maintainability: Corrective maintenance cannot avoid the risk of failure, and regular maintenance may lead potential risk of additional repair. But PHM can promote the development of CBM [3]. It tries its best to reduce unplanned maintenance, to improve maintenance efficiency and to economize maintenance costs.

4) Supportability: The scale of aviation support is large, which contains a number of discrete parts and complex support devices. PHM can management support activities, optimize support resources and cut support scale [4].

5) Testability: The problems of CND (cannot duplicate) and RTOK (re-test OK) in BIT (built in test) cannot be resolved [5]. Through the real-time monitor, sensors collect lots of information about system and its actual processing environment and condition. And using this information in prognostic model can reduce the false alarm rate.

6) Economy: The primary goal of PHM is economic affordability. PHM has a huge economic advantage in reducing the cost of normal test, spare parts, maintenance 
scale, unnecessary downtime and manpower and in making full use of products' life span [6].

\section{FRAMEWORK OF COMPLEX ELECTROMECHANICAL EQUIPMENT}

In order to study the application of PHM for complex electromechanical equipment, the monitor parameters should be identified first, which include operating parameters and state parameters [7]. The principle of parameters selection is to be all-around and easy to get. Being all-around means the selected parameters can comprehensively reflect the state and trend of complex electromechanical equipment, and being easy to get means the selected parameters can be easily collected and processed. The parameters selection can be conducted by FMECA [8]. The framework of PHM for complex electromechanical equipment is built based on monitoring the selected parameters, which shows in Fig.2.

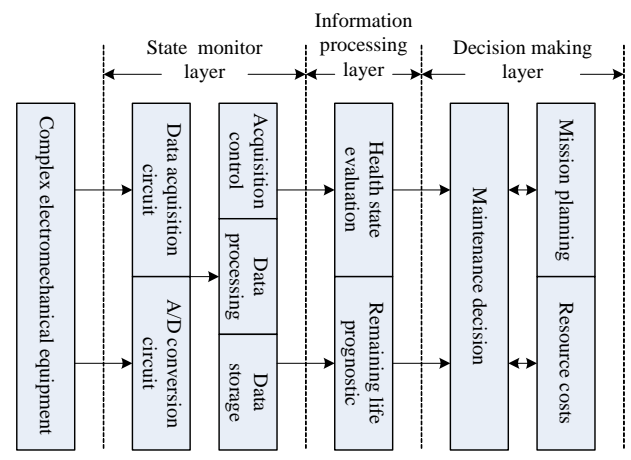

Figure 2. PHM framework of complex electromechanical equipment

State monitoring layer fulfill data acquisition, including data processing and data storage. Information processing layer use acquired and historically stored data to evaluate health state and predict remaining life. Decision making layer can perform health management and give maintenance decision utilizing the output information from information processing layer combined with tasks and available resources.

\section{A. Design of state mornitoring layer}

The major assignment of state monitoring layer is to acquire the state data of electromechanical equipment. Its basic design includes hardware design and software design.

1) Hardware design

The hardware of complex electromechanical equipment is mainly composed of parameters acquisition circuits, $A / D$ switching circuits and control computers.

Parameters acquisition circuits need to identify the type, accuracy, bandwidth and optimized layout of sensors according to the selected state monitoring parameters.

A/D switching circuits include signal conditioning circuits and A/D sampling circuits. Signal conditioning circuits are used to process signals collected by parameters acquisition circuits in order to meet input requirements of A/D sampling circuits. Nowadays mature acquisition modules are usually applied in A/D sampling circuits, which have features of many acquisition channels, short collecting process, high resolution ratio.
With the help of built-in state monitoring software, control computers take control of state data collecting, data pre-processing and data storage.

2) Software design

State monitoring layer software mainly executes functions of collection control, data pre-processing and data storage. Fig. 3 shows the basic function modules.

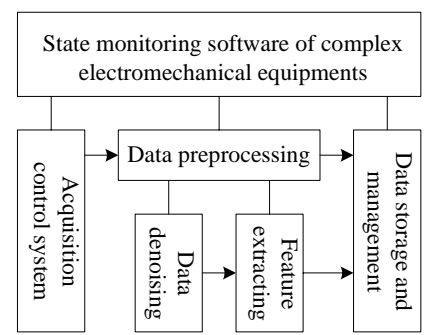

Figure 3. Basic function modules of state monitoring software

Collection control mainly accomplishes the collection of specified parameters. Its program is compiled through LabWindows/CVI.

Data pre-processing mainly complete pre-processing of the data collected, mainly including data denoising and feature extracting.

Data denoising detects and eliminates based on $53 \mathrm{H}$ method abnormal value. The basic idea is to compare measurement with a smooth curve estimate which is generated before, for identifying outliers [9].

Feature parameters of Complex electromechanical equipment contain deterministic signals such as voltage and current and stochastic signals such as vibration and excursion. Statistics, for instance mean and variance, can be used to describe deterministic signals. And feature extraction is required for stochastic ones.Based on the ARMA model, the system analyzes stochastic signals such as vibration by using fitted parameters of the model. The modeling procedure is demonstrated in Fig. 4 [10].

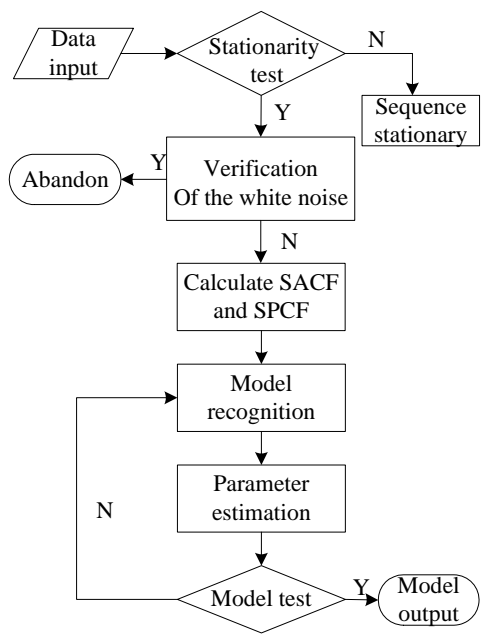

Figure 4. Modeling procedure based on ARMA 
After several processes such as stationary test, white noise test, model identification, model order determination, model parameters evaluation and validity test, the ARMA model is then as follows (1).

$$
\begin{aligned}
x_{t}= & \varphi_{1} x_{t-1}+\varphi_{2} x_{t-2}+\cdots \varphi_{p} x_{t-p}+\varepsilon_{t} \\
& -\theta_{1} \varepsilon_{t-1}-\theta_{2} \varepsilon_{t-2}-\cdots-\theta_{q} \varepsilon_{t-q}
\end{aligned}
$$

Where $\left\{\varphi_{1}, \varphi_{2}, \cdots, \varphi_{p}\right\}$ are auto-regression parameters, $\left\{\theta_{1}, \theta_{2}, \cdots, \theta_{q}\right\}$ are glide mean parameters, and $\varepsilon_{t}$ is the stagger. For they contain random sequences' information of different states monitoring, these parameters can be used as eigenvectors to describe stochastic signals in different states.

Storage of monitoring data and eigenvectors is achieved by state data storage and management, which also stores measurand's information, such as the equipments' device number, and monitoring information such as monitoring time. It can also conduct management by utilizing database, thus making it possible to observe and call when subsequent information is processed.

\section{B. Design of information processing layer}

Information processing layer is playing a connecting role in prognostic and health management of complex electromechanical equipment. Its main mission is to make an evaluation of the equipment's present health state and a prognostic of the remaining life.

1) Health state evaluation

Health state evaluation helps to identify the degree of equipment's deviation from normal state. Health state degradation of complex electromechanical equipment is continuous. Traditionally its health state is divided into normal and fault state by fault threshold. For the purpose of on-condition maintenance, many degraded states between normal states and fault ones are divided detailed whose diversity indicates different extent of degradation. In order to train model identification, health state evaluation needs to acquire data of different degraded states in whole life circle. The identification model combined with present state monitoring data can help to evaluate complex electromechanical equipment's current health state.

With simple structure and strong compliance, BP neural network has a nice classification capacity of arbitrary shape, thus achieving wide application and becoming the most mature network model so far [11].

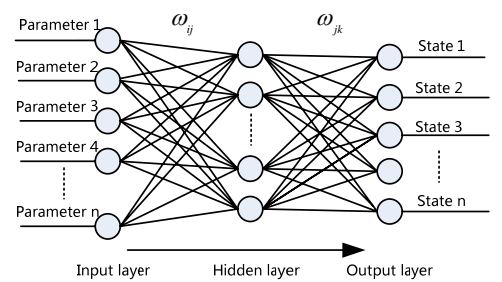

Figure 5. State recognition model of BP neural network

Using BP neural network shown in Fig.5 as health state identification model, the system divides complex electrome- chanical equipment's health state into $\mathrm{N}$ classes, which represent normal state, different degraded states and fault state respectively. Train BP neural network based on monitoring data in different state. On the basis of trained BP neural network, health state evaluation according to the given state monitoring data can be achieved.

2) Remaining life prognostic

Remaining life is a time span from the start of detection to the point when the equipment breaks down. Remaining life prediction is an important basis for equipment's maintenance, replacement and spare parts' strategy formulation. There are traditional prediction models and methods such as prediction based on statistics, time series prediction, neural network prediction and Bayesian network prediction [12] [13].

Introducing AGO (Accumulated generating operation) into support vector regression, the system proposes a complex electromechanical equipment grey prediction model of remaining life combination, which supports vector. Fig.6 shows the basic structure of the model.

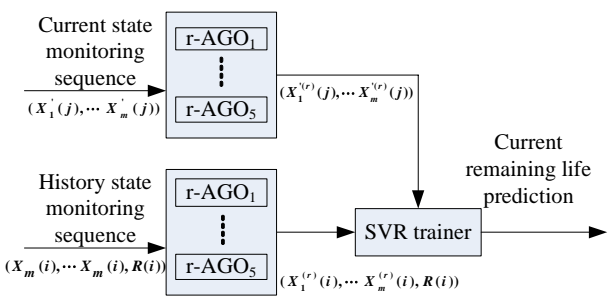

Figure 6. Remaining life prediction model of grey SVM

First, conduct accumulated generating operation for time series of original data (including monitoring sequences of history state and current state). For instance, conduct $r$ times accumulated generating operation for some parameter's original data sequence $X^{0}$ (denoted by $r$-AGO) and obtain $r$ order accumulated generating data sequence $X^{r}$, as in (2).

$$
x^{r}(k)=\sum_{i=1}^{k} x^{r-1}(i), \quad k=1, \cdots, n
$$

Support vector regression (SVR) is an intellectual regression method based on the theory of interval maximization and structural risk minimization. With high generalization, it can help to process nonlinear and highdimensional data of small sample and overcome local minima. For nonlinear regression estimation, SVR builds regression estimation model as follows (3) by mapping sample space to high dimensional feature space based on nonlinear mapping $\Phi\left(x_{i}\right)$.

$$
\begin{aligned}
& \min \frac{1}{2}\|\omega\|^{2}+C \sum_{i=1}^{l}\left(\xi_{i}+\xi_{i}^{*}\right) \\
& \text { s.t. }\left\langle\omega, \Phi\left(x_{i}\right)\right\rangle+b-y_{i}<\xi_{i}+\varepsilon \\
& y_{i}-\left\langle\omega, \Phi\left(x_{i}\right)\right\rangle-b<\xi_{i}^{*}+\varepsilon \\
& \quad \xi_{i}, \xi_{i}^{*} \geq 0, \quad i=1,2, \cdots, l
\end{aligned}
$$


Where $\mathrm{C}$ is penalty parameter, $\xi_{i}, \xi_{i}^{*}$ are slack variable and $\varepsilon$ is insensitive loss metric. (3) shows a typical quadratic programming problem, whose dual problem is obtained by using Lagrange product vector. Its solution model, namely regression model of life prediction, shows in (4).

$$
\begin{aligned}
R U L(X) & =\sum_{j=1}^{l}\left(a_{j}-a_{j}^{*}\right) \Phi\left(x_{j}\right) \cdot \Phi(x)+b \\
& =\sum_{j=1}^{l}\left(a_{j}-a_{j}^{*}\right) K\left(x_{j}, x\right)+b
\end{aligned}
$$

Where $a_{j}, a_{j}^{*}$ are multiplying coefficients and $K\left(x_{j}, x\right)$ is the kernel function which meets the Mercer condition.

The remaining life of complex electromechanical equipment can be predicted by using trained regression to process current monitoring data after $r$ times accumulated generating operation and to run regression analysis.

\section{Design of decision making layer}

Decision making layer is the foothold of the entire prediction and health management system, whose main mission is to utilize information from information processing layer combined with mission requirements and resources. Thus it can reduce support cost and enhance system availability in the meantime. Fig.7 shows the function modules of decision making layer which mainly contains information management module, maintenance decision module and resources management module.

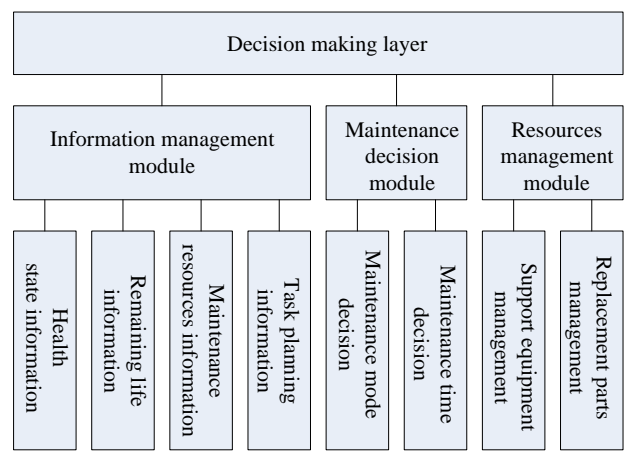

Figure 7. Function modules of decision making layer

Information management module is to effectively manage health state information, remaining life information, maintenance resources information and task planning information in order to provide decision-making basis for maintenance support.

Maintenance decision module is to make scientific and reasonable decision for maintenance mode and time of the equipment based on information from information management module, ensuring the maximum satisfaction of the equipment's utilization requirements and economically affordable goal.
Resources management module is mainly to simplify support resources scale, reduce resources deployment investment and decrease assurance cost according to information from information module combined with resources such as dynamic programming support equipment and replacement parts on the precise of meeting requirements of peacetime and wartime task.

\section{MODEL VERIFICATION}

PHM is verified by its application for DTG (Dynamically Tuned Gyroscope). Five operational parameters of DTG by FMMEA, including vibration, temperature, random shift, motor power and power supply voltage, are chosen as monitoring parameters and three DTGs have been under long term monitoring research. And eigenvector is obtained by fitting analysis of two random variables vibration and shift utilizing ARMA model, a total of 386 sets of monitoring data from normal state to fault state. The method of health state evaluation and remaining life prediction is analyzed and verified according to these data.

\section{A. Analysis of health state evaluation}

Health state evaluation of the data is conducted based on BP neural network. Single hidden layer BP network is adopted with 12 import cells, 3 output cells and 11 interface layer cells, where 001 represents normal state, 010 represents degraded state and 100 represents fault state. Suppose learning rate is 0.8 and error of single sample is $10-4$. After fully training on the network based on 306 sets of data, the network is tested with the rest 80 sets and (Z, Y, X) represents output of each sample point. Fig. 8 shows output distributions of 80 samples and Tab.1 shows the test result.

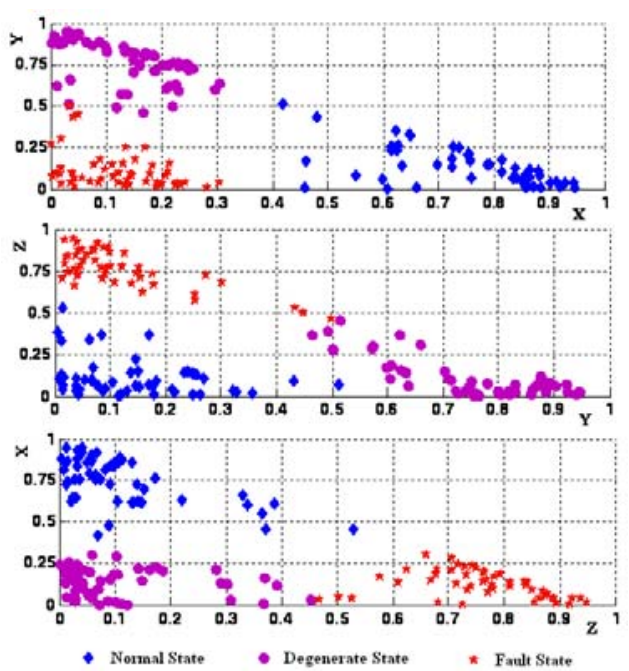

Figure 8. Tested samples output distributions

TABLE I. Prediction Result OF BP NEURAL NetWork

\begin{tabular}{cccc}
\hline State & Normal & Degraded & Fault \\
\hline Neural network code & 001 & 010 & 100 \\
\hline Recognition rate & $92 \%$ & $96 \%$ & $98 \%$ \\
\hline
\end{tabular}


BP neural network shows good ability of recognizing the state of DTG both in Fig. 8 and Tab. 1.

\section{B. Analysis of remaining life prediction}

Remaining life of the grey SVM regression model is predicted according to 58 sets of monitoring data of one DTG from normal state to fault state. Leave-one-out method is used to analyze, which uses one set as test data and the rest as train data. Analysis results are shown in Fig.9.

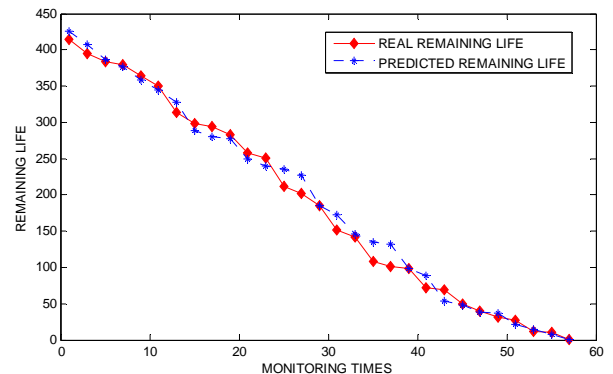

Figure 9. Contrast between real remaining life and predicted one

Statistical analysis suggests that, the average relative error of prediction is $4.3 \%$ and the maximum relative error is $15.6 \%$. The result of prediction analysis indicates a high degree of accuracy of the grey SVM applied in this paper.

\section{CONCLUSION}

With the rapid development of PHM, each aspect of its technical theory has been widely applied. However as a systematic interdiscipline, seldom has system application or engineering practice of PHM been achieved. In this paper, PHM's application in systematic design and applied research of complex electromechanical equipment is studied in condition monitoring, information processing and health management three aspects. At present, the system has been applied in many DTGs, and practical application indicates that this system can reasonably reflect DTG's health state and remaining life. Based on decision making layer, it can effectively manage maintenance activities and resources, enhance the task availability of DTG and reduce support costs. Moreover, the design of this system can also provide great significance for the prediction of PHM's application to other objects.

\section{REFERENCES}

[1] Hess. Andrew, Calvello.G, and Dabney.T, "PHM a Key Enabler for the JSF Autonomic Logistics Support Concept,” Aerospace Conference 2004 Proceedings, 2004.

[2] Bo Sun, Shengkui Zeng, and Rui Kang, "Benefits Analysis of Prognostics in Systems,” 2010 Prognostics \& System Health Management Conference, 2010.

[3] Z.M.Yang, D.Djurdjanovic, and J.Ni, "Maintenance schedul-ing in manufacturing systems based on predicted machine degradation, Journal of Intelligent Manufacturing, vol.19, Jan.2008, pp. 87-98.

[4] S.L.Dreyer, "Autonomic logistic-Developing an implementa-tion approach for an existing military weapon system," IEEE Instrumentation and Measurement Magazine, vol.9, Apr.2006, pp. 16-21.

[5] R. Williams, J.Banner, and I.Knowlis, "An investigation of 'cannot duplicate'failures,” Quality and Reliability Engineering International, vol.14, May.1998, pp. 331-337.

[6] E.Scanff, K.L.Feldman, and S.Ghelam, "Life Cycle cost impact of using prognostic and health management for helicopter avionics," Microelectronics Reliability, vol.47, Dec.2007, pp. 1857-1864.

[7] N.Vichare, P. Rodger, and V.Eveloy, "Environment and usage monitoring of electronic products for health assessment and product design,” International Journal of Quality Technology and Quantitative Management, vol.4, Feb.2007, pp. 235-250.

[8] J.Gu and M.Pecht, "Porgnostics and health management using physics-of-failure," 54th Annual Reliability \& Maintainability Symposium, 2008.

[9] Z.K.Peng and F.L.Chu, "Application of the wavelet transform in machine condition monitoring and fault diagnostics: A review with bibliography," Mechanical System and Signal Processing, vol.18, Feb.2004, pp. 199-221.

[10] Cao Xinyan and Zou Yingyong, "A research on the cross spectrum method for determining the weak signal frequency estimation in colored noise," Journal of Changchun University, vol.20, Jun.2010, pp. 52-55.

[11] Xiaoyu Zhang, Guifen Cai, "Application of BP Neural Network in the Fault Prediction for a Certain Aero Motor," Development\&Innovation of Machinery\&Electrical Products, vol.21, Feb.2008, pp. 53-55.

[12] G. Pulcini, “An exponential reliability-growth model in multicopy tesing program,” IEEE Transactions on Reliability, vol.50, Apr.2001, pp. 365-373.

[13] J.I.Yeh and L.S.Mao, "Prediction of membrane proteins in Mycobacterium tuberculosis using a Support Vector Machine algorithm,” Journal of Computation Biology, vol.13, Jan. 2006, pp. 126-129 